\title{
Long-Term Performance of Right Ventricular Implantable Cardioverter-Defibrillator Leads in Arrhythmogenic Right Ven- tricular Cardiomyopathy and Hypertrophic Cardiomyopathy
}

\author{
Yuichiro Sagawa, ${ }^{1}$ MD, Yasutoshi Nagata, ${ }^{1}$ MD, Tetsuo Yamaguchi, ${ }^{2}$ MD, Kentaro Mitsui, ${ }^{1}$ MD, \\ Tatsuhiro Nagamine, ${ }^{1}$ MD, Junji Yamaguchi, ${ }^{1}$ MD, Sadahiro Hijikata, ${ }^{1}$ MD, Keita Watanabe, ${ }^{1}$ MD, \\ Ryo Masuda, ${ }^{1}$ MD, Ryoichi Miyazaki, ${ }^{1}$ MD, Masakazu Kaneko, ${ }^{1}$ MD, Naoyuki Miwa, ${ }^{1}$ MD, \\ Masahiro Sekigawa, ${ }^{1}$ MD, Nobuhiro Hara, ${ }^{1}$ MD, Toshihiro Nozato, ${ }^{1}$ MD, Takashi Ashikaga, ${ }^{1}$ MD, \\ Masahiko Goya, ${ }^{3} \mathrm{MD}$, Tetsuo Sasano, ${ }^{3} \mathrm{MD}$ and Kenzo Hirao, ${ }^{4} \mathrm{MD}$
}

\begin{abstract}
Summary
Patients with arrhythmogenic right ventricular cardiomyopathy (ARVC) and hypertrophic cardiomyopathy (HCM) implanted with implantable cardioverter-defibrillators (ICDs) may show a large decrease in R-wave amplitude during long-term follow-up. However, it is unclear whether this decrease is higher in these patients than in those without structural heart disease. This study investigated ICD-lead intracardiac parameters over a long duration in patients with ARVC and HCM and compared these parameters with those of a control group. We included 50 patients (mean age, $55.2 \pm 17.2$ years; $26 \%$ female) with ICD leads in the right ventricular apex, and compared 7 ARVC and 14 HCM patients with 29 control patients without structural heart disease. ICD-lead parameters, including R-wave amplitude, pacing threshold, and impedance during follow-up, were compared. The difference in these parameters between the time of implantation and year 5 were also compared. There were no significant differences in R-wave amplitude at implantation among the 3 groups. The change in R-wave amplitude between the time of implantation and year 5 was significantly greater in the ARVC group $(-3.3 \pm 5.4 \mathrm{mV}$, $P=0.012)$ in comparison to the control group $(1.3 \pm 2.8 \mathrm{mV})$; the $\mathrm{HCM}$ group showed no significant difference $(-0.4 \pm 2.3 \mathrm{mV}, P=0.06)$. Thus, in the ARVC group, R-wave amplitude at year 5 was significantly lower than that in the control group $(5.7 \pm 4.8 \mathrm{mV}$ versus $12.5 \pm 4.5 \mathrm{mV}, P=0.001)$. In ARVC patients with ICDs, ventricular sensing is likely to deteriorate during long-term follow-up; however, in HCM patients, sensing may not deteriorate.
\end{abstract}

(Int Heart J 2020; 61: 39-45)

Key words: Shock lead, Intracardiac parameters, Decrease in R-wave amplitude

I mplantable cardioverter-defibrillators (ICDs) are used to treat ventricular arrhythmias (VA) and to reduce the risk of sudden cardiac death. ${ }^{1,2)}$ The amplitude of the intracardiac R-wave is crucial for the performance of ICDs. Poor sensing and a decrease in R-wave amplitude may result in VA undersensing, inducing inappropriate pacing, or preventing optimal defibrillator therapy. ${ }^{3,4}$ Thus, the long-term performance of the ICD leads should be carefully monitored for the safety of patients with ICDs. In particular, patients with progressive diseases such as arrhythmogenic right ventricular cardiomyopathy (ARVC) need to be followed up more carefully. Previous studies have reported that a decrease in $\mathrm{R}$-wave amplitude was high in patients with ARVC and hypertrophic cardiomyopathy (HCM) who were implanted with ICDs during long-term follow-up. ${ }^{4,5)}$ ARVC is a progressive cardio- myopathy, and the region known as the "triangle of dysplasia" in the right ventricle is replaced with fibrofatty tissue. $^{6-8)}$ The right ventricular (RV) apex is within the triangle of dysplasia and is the typical target for ICD-lead placement. Hence, the intracardiac parameters of ICD leads implanted in the RV apex in ARVC patients are likely to deteriorate during long-term follow-up. ${ }^{5)}$ In contrast, $\mathrm{HCM}$ is a cardiomyopathy that causes abnormalities in the left ventricle and is generally not a progressive disease, except in some cases, where it progresses to dilatedphase HCM. These cases are characterized by left ventricular systolic dysfunction and cavity dilatation when followed up over a long period. ${ }^{9-11)}$ Accordingly, we hypothesized that the decrease in R-wave amplitude would be higher in patients with ARVC than in control patients and that there would be no difference in the decrease in

From the 'Department of Cardiology, Japanese Red Cross Musashino Hospital, Tokyo, Japan, ${ }^{2}$ Department of Cardiovascular Center, Toranomon Hospital, Tokyo, Japan, ${ }^{3}$ Department of Cardiovascular Medicine, Tokyo Medical and Dental University, Tokyo, Japan and ${ }^{4}$ Arrhythmia Advanced Therapy Center, AOI Universal Hospital, Kanagawa, Japan.

Address for correspondence: Tetsuo Yamaguchi, MD, Department of Cardiovascular Center, Toranomon Hospital, 2-2-2, Toranomon, Minato-ku, Tokyo 1058470, Japan. E-mail: tetsuo5672@yahoo.co.jp

Received for publication May 30, 2019. Revised and accepted October 7, 2019

Released in advance online on J-STAGE January 17, 2020.

doi: 10.1536/ihj.19-279

All rights reserved by the International Heart Journal Association. 


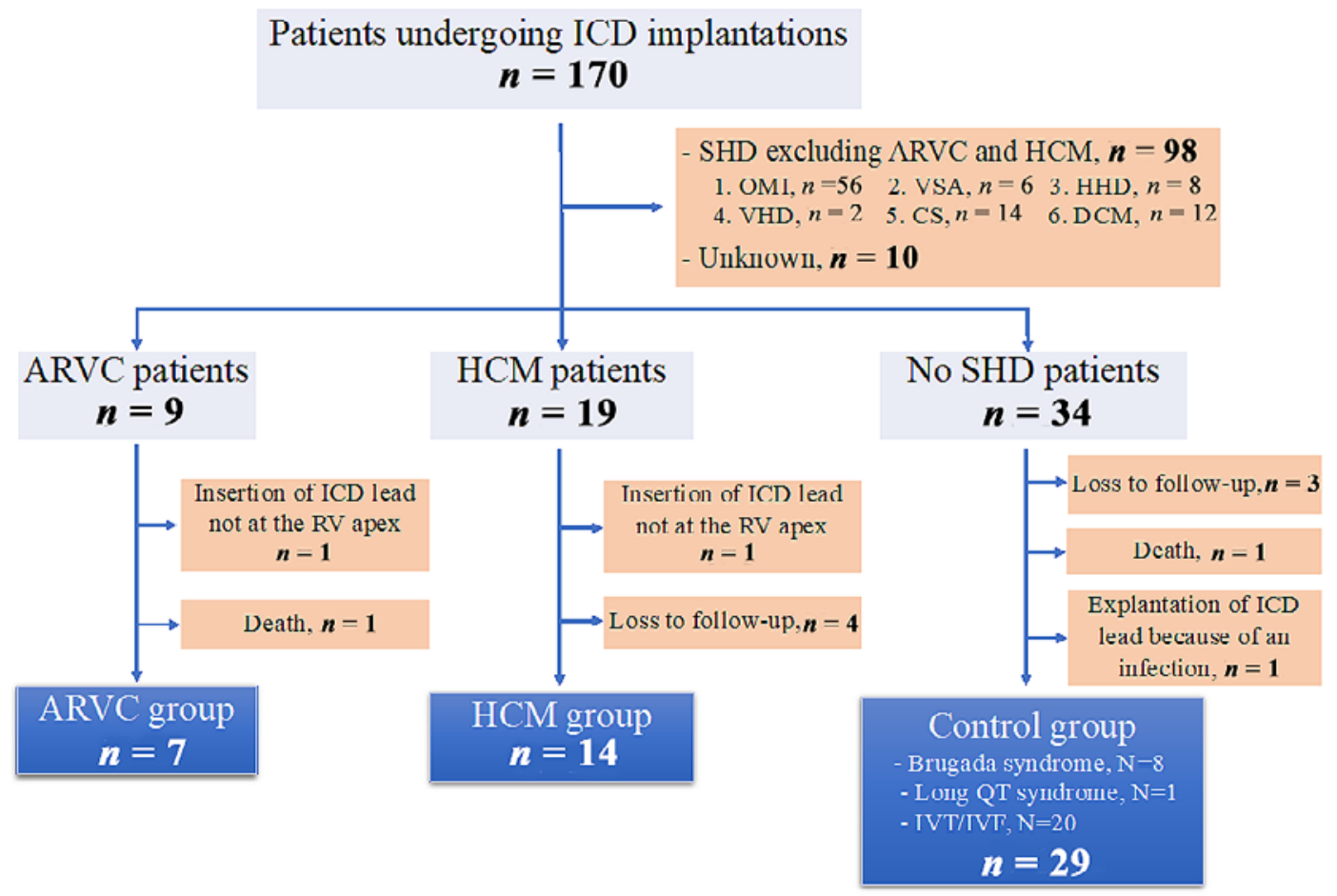

Figure 1. Schematic summary of the study flowchart. ICD indicates implantable cardioverter-defibrillator; SHD, structural heart disease; ARVC, arrhythmogenic right ventricular cardiomyopathy; HCM, hypertrophic cardiomyopathy; OMI, old myocardial infarction; VSA, vasospastic angina; HHD, hypertensive heart disease; VHD, valvular heart disease; CS, cardiac sarcoidosis; DCM, dilated cardiomyopathy; IVF, idiopathic ventricular fibrillation; IVT, idiopathic ventricular tachycardia; and $\mathrm{RV}$, right ventricular.

R-wave amplitude between HCM and control patients because HCM does not usually involve the right ventricle. The purpose of this study was to investigate the intracardiac parameters of ICD leads implanted in the RV apex over the long term in patients with ARVC and HCM. Moreover, we aimed to compare the parameters of ARVC and HCM with those of a control group.

\section{Methods}

Study patients: This retrospective study identified 170 consecutive patients who underwent an ICD device implantation from December 1998 to March 2014 at the Japanese Red Cross Musashino Hospital. Ninety-eight patients with structural heart disease (SHD), excluding ARVC and HCM, and 10 patients whose underlying disease was unknown were excluded. Nine patients with ARVC, 19 with HCM, and 34 controls without SHD were identified. Further, patients with any of the following conditions were also excluded: (1) an ICD lead placed in a location other than the RV apex; (2) an ICD-lead explantation because of an infection during the follow-up period; (3) death during the follow-up period; and (4) loss to follow-up. Finally, 50 patients (7 patients with ARVC, 14 with HCM, and 29 control patients) were included in the current analysis. A summary of the study flow is shown in Figure 1. The diagnosis of ARVC was confirmed using the original 1994 or 2010 Task Force Criteria. ${ }^{12,13)}$ The diagnosis of HCM required the demonstration of a hypertro- phied left ventricle, which was identified by twodimensional echocardiography and/or cardiac magnetic resonance imaging. ${ }^{14-16)}$ The control group consisted of patients without SHD, including Brugada syndrome, long QT syndrome, and idiopathic ventricular fibrillation or tachycardia. This study was approved by the institutional review board of the Japanese Red Cross Musashino Hospital and complied with the ethical principles of the Declaration of Helsinki and the Japanese Ethical Guideline for Medical and Health Research Involving Human Subjects. All participants were notified regarding their inclusion in the study, and we explained to them that they were free to opt out of participation at any time (ethical approval number 30030).

Baseline characteristics and parameters of the ICD leads: We collected baseline data regarding information on demographics (age and sex), comorbidities, echocardiographic parameters, brain natriuretic peptide levels, and parameters of the ICD leads, including R-wave amplitude, pacing threshold, and pacing impedance.

Follow-up strategy: All patients routinely underwent a device interrogation at the time of implantation and 2 or 3 days after implantation. Follow-up was scheduled for 3 months after discharge and then every 3 to 6 months at our institution. This study involved a follow-up for 5 years after ICD implantation, and the ICD-lead parameters (R-wave amplitude, pacing threshold, and pacing impedance) during follow-up were compared between the ARVC and control groups and between the HCM and 
Table I. Baseline Clinical Characteristics

\begin{tabular}{|c|c|c|c|c|c|}
\hline & $\begin{array}{l}\text { Total patients } \\
\quad(n=50)\end{array}$ & $\begin{array}{l}\text { ARVC } \\
(n=7)\end{array}$ & $\begin{array}{l}\text { HCM } \\
(n=14)\end{array}$ & $\begin{array}{l}\text { Control } \\
(n=29)\end{array}$ & $P$-value \\
\hline Age, years & $55.2 \pm 17.2$ & $48.9 \pm 16.9$ & $65.2 \pm 10.9$ & $51.9 \pm 18.1$ & 0.019 \\
\hline Female, $n(\%)$ & $13(26)$ & $1(14)$ & $6(43)$ & $6(21)$ & 0.238 \\
\hline Hypertension, $n(\%)$ & $11(22)$ & $0(0)$ & $5(36)$ & $6(21)$ & 0.088 \\
\hline Diabetes, $n(\%)$ & $4(8)$ & $0(0)$ & $3(21)$ & $1(3)$ & 0.099 \\
\hline Chronic kidney disease, $n(\%)$ & $5(10)$ & $0(0)$ & $4(29)$ & $1(3)$ & 0.029 \\
\hline \multicolumn{6}{|l|}{ Echocardiography } \\
\hline Ejection Fraction $(\%)$ & $66.1 \pm 9.8$ & $62.9 \pm 8.9$ & $71.2 \pm 9.4$ & $64.3 \pm 9.5$ & 0.062 \\
\hline $\mathrm{BNP}(\mathrm{pg} / \mathrm{mL})$ & $55.6(14.8-179.7)$ & $45.1(22.5-153)$ & $204(158.7-588.6)$ & $17(5.8-65.1)$ & $<0.001$ \\
\hline Dilated-phase HCM, $n(\%)$ & & & $2(14)$ & & \\
\hline \multicolumn{6}{|l|}{ Diagnosis } \\
\hline Brugada syndrome, $n(\%)$ & & & & $8(28)$ & \\
\hline Long QT syndrome, $n(\%)$ & & & & $1(3)$ & \\
\hline Idiopathic ventricular fibrillation/tachycardia, $n(\%)$ & & & & $20(69)$ & \\
\hline
\end{tabular}

ARVC indicates arrhythmogenic right ventricular cardiomyopathy; HCM, hypertrophic cardiomyopathy; and BNP, brain natriuretic peptide.

control groups. Moreover, the differences between these parameters at the time of implantation and at year 5 (between 48 and 60 months after the ICD implantation) were also compared between the ARVC and control groups and between the HCM and control groups.

Lead location: In this study, only patients with the ICD lead implanted in the RV apex were included. In all patients, the ICD-lead positioning was assessed via radiography, fluoroscopy, or computed tomography, and the lead location was analyzed with reference to the reports of previous studies. ${ }^{17,18)}$ Electrocardiographic findings were also referenced when it was difficult to determine the lead location. Two independent readers (YS, TY) determined the lead locations. If the two readers did not agree on the lead location, a third reader (YN) determined the lead location. Lead failure: Lead failure was defined as a loss of capture or sudden rise in thresholds; recurrent nonphysiological high-rate sensing; a sudden change in lead impedance, unrelated to perforation or lead dislodgement; or abnormal lead parameters associated with an evident lead fracture or insulation failure. In this study, lead failure did not occur in any patient during the entire followup.

Statistical analysis: Categorical variables are summarized as numbers and percentages and continuous variables as the mean \pm standard deviation or median and interquartile range. The differences in clinical characteristics among the 3 groups were analyzed using one-way analysis of variance test, the Kruskal-Wallis test, or chi-squared test, as appropriate. A comparison of the ICD-lead parameters was conducted using the unpaired t-test or Mann-Whitney U-test, as appropriate. All analyses were performed with JMP software version 12.2.0 (SAS Institute Inc., Cary, NC, USA). A two-sided $P$-value $<0.05$ was considered statistically significant.

\section{Results}

Patient characteristics: The baseline characteristics of the patients are summarized in Table I. In the ARVC group, the mean age was $48.9 \pm 16.9$ years and the patients had no comorbidities. In the HCM group, the mean age was $65.2 \pm 10.9$ years and there were two patients with dilated-phase HCM. Among the 3 groups, the HCM patients were the oldest and had the highest ratio of chronic kidney disease and highest brain natriuretic peptide level.

ICD device implantation: The ICD device was implanted subcutaneously with a transvenous lead system. Fortyseven procedures $(94 \%)$ were left-sided, and 33 dualchamber $(66 \%)$ ICDs were implanted. In all patients, the ICD lead was implanted in the RV apex. Thirteen active $(26 \%)$ and 37 passive (74\%) fixation leads were used, and 9 integrated bipolar leads $(18 \%)$ were selected in this study.

The trend in ICD lead parameters during the followup: No significant difference was noted in any of the intracardiac parameters at the time of implantation between the ARVC and control groups. After a plateau of the Rwave amplitude in the first 3 years, a sharp decrease between year 3 and year 5 was observed in the ARVC group. However, in the HCM and control groups, the Rwave amplitude did not markedly change during the entire follow-up. Thus, in the ARVC group, the R-wave amplitude at year 5 was significantly lower than that in the control group $(5.7 \pm 4.8 \mathrm{mV}$ versus $12.5 \pm 4.5 \mathrm{mV}, P=$ 0.001 ), but no significant difference was seen in the Rwave amplitude at year 5 between the HCM and control groups. The ARVC group had a sharp increase in the pacing threshold in the first year, and then the pacing threshold plateaued during the follow-up. In the ARVC group, the pacing threshold from year 1 to year 5 was significantly higher than that in the control group. The pacing impedance tended to decrease during the follow-up in all 3 groups, and no significant difference was seen at year 5 between the ARVC and control groups and between the HCM and control groups (Figure 2).

The decrease in R-wave amplitude between the time of implantation and year 5 was significantly greater in the ARVC group than in the control group $(-3.3 \pm 5.4 \mathrm{mV}$ versus $1.3 \pm 2.8 \mathrm{mV}, P=0.012)$. In contrast, the amount of change in R-wave amplitude was similar between the $\mathrm{HCM}$ and control groups $(-0.4 \pm 2.3 \mathrm{mV}$ versus $1.3 \pm 2.8$ $\mathrm{mV}, P=0.06)$. Regarding the amount of change in pacing 

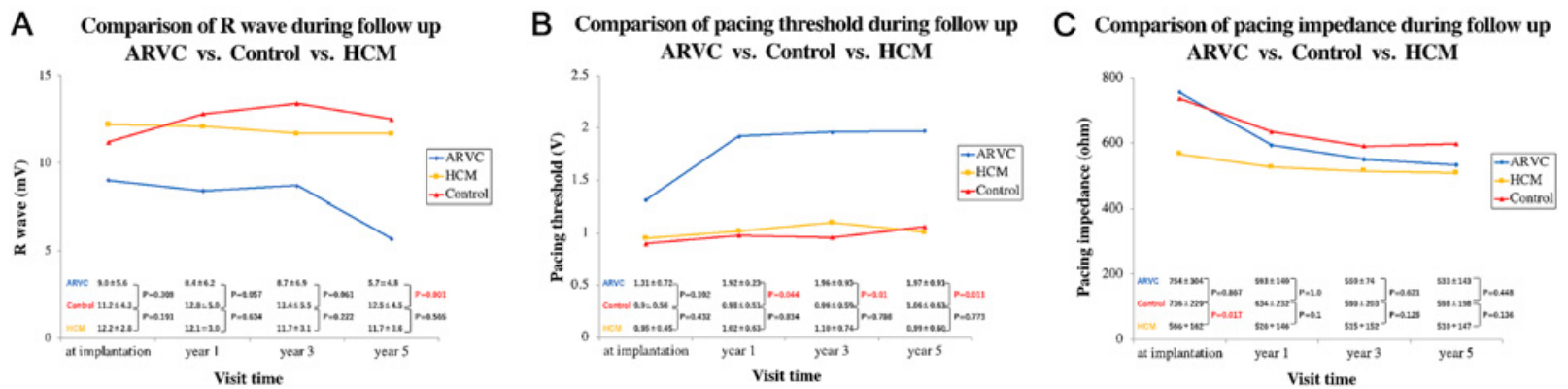

Figure 2. Comparison of intracardiac parameters during follow-up. A: The trend of R-wave amplitudes during follow-up among the ARVC, HCM, and control groups. In the ARVC group, after a plateau of the R-wave amplitude in the first 3 years, a sharp decrease between year 3 and year 5 was observed, and the R-wave amplitude at year 5 was significantly lower than that in the control group. B: The trend of pacing thresholds during follow-up. In the ARVC group, after a sharp increase in the first year, the pacing threshold remained flat during follow-up. In the ARVC group, the pacing threshold from year 1 to year 5 was significantly higher than that in the control group. C: The trend of pacing impedances during follow-up. In all 3 groups, the pacing impedance tended to decrease during the entire follow-up, but no significant difference was seen among the ARVC, HCM, and control groups. ICD indicates implantable cardioverter-defibrillator; ARVC, arrhythmogenic right ventricular cardiomyopathy; and HCM, hypertrophic cardiomyopathy.

A

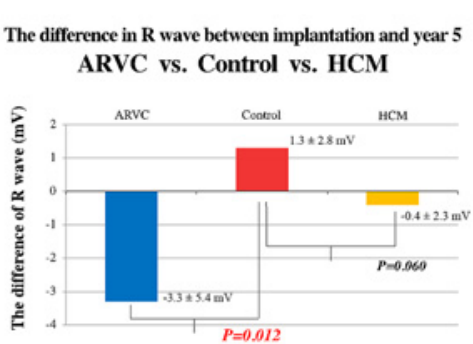

B

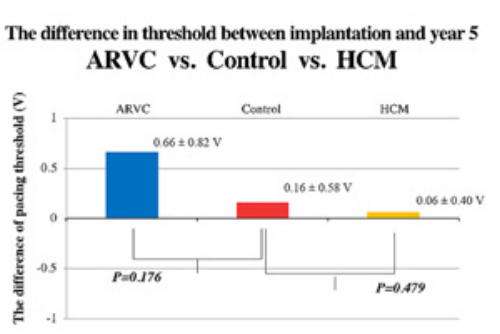

C

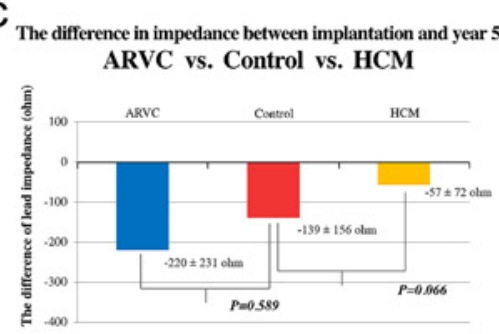

Figure 3. Difference in intracardiac parameters between implantation and year $5 \mathrm{~A}$ : The decrease in R-wave amplitude between the time of implantation and year 5 among the ARVC, HCM, and control groups. The decrease was significantly greater in the ARVC group than in the control group. B, C: Respectively, the amount of change in pacing thresholds and pacing impedances between the time of implantation and year 5 among the 3 groups. No significant difference was observed in these parameters among the 3 groups. ICD indicates implantable cardioverter-defibrillator; ARVC, arrhythmogenic right ventricular cardiomyopathy; and HCM, hypertrophic cardiomyopathy.

Table II. Patient Characteristics and Trend of R-wave Amplitude during Follow-up in the ARVC Group

\begin{tabular}{ccccccccc}
\hline $\begin{array}{c}\text { Patient } \\
\text { No. }\end{array}$ & $\begin{array}{c}\text { Age } \\
(\text { years })\end{array}$ & Sex & $\begin{array}{c}\text { Echocardiography } \\
\text { LVEF } \\
(\%)\end{array}$ & $\begin{array}{c}\text { RVD } \\
(\mathrm{mm})\end{array}$ & Implantation & Year 1 & Year 3 & Year 5 \\
\hline 1 & 52 & Male & 70 & 43 & 16.9 & 5 & 4 & 2.6 \\
2 & 61 & Male & 70 & 37 & 3 & 4 & 4 & 4 \\
3 & 65 & Male & 60 & 32 & 5 & 5 & 5 & 5 \\
4 & 14 & Male & 67 & 35 & 9.7 & 12 & 12 & 3.3 \\
5 & 52 & Male & 67.4 & 32 & 3 & 1 & 1 & 1 \\
6 & 55 & Male & 61 & 27 & 15.2 & 15.2 & 15.2 & 15.2 \\
7 & 43 & Female & 45 & 32 & 10.4 & 16.9 & 19.4 & 8.8 \\
\hline
\end{tabular}

ARVC indicates arrhythmogenic right ventricular cardiomyopathy; LVEF, left ventricular ejection fraction; and RVD, right ventricular diameter.

threshold and pacing impedance, the ARVC and HCM groups did not significantly differ from the control group (Figure 3). In the ARVC group, patients who showed decline in $\mathrm{R}$ wave demonstrated further RV enlargement compared with those who did not, but there was no statistically significant difference (RV diameter; $36 \pm 5 \mathrm{~mm}$ versus $32 \pm 5 \mathrm{~mm}, P=0.41)$. The patient characteristics and the trend of R-wave amplitude during follow-up in each ARVC patient are summarized in Table II.
Undersensing of VA associated with a decrease in the R-wave: In this study, in one patient with ARVC, the Rwave amplitude decreased from $3 \mathrm{mV}$ to $1 \mathrm{mV}$ at 5 years after implantation. Undersensing of the $\mathrm{R}$ wave caused the delay in a proper shock during the defibrillation test. Therefore, we implanted a new ventricular lead in the RV septum. After the re-implantation, no further decrease in R-wave amplitude occurred over the course of 2 years. 


\section{Discussion}

The main findings of this study were as follows: (1) regarding the $\mathrm{R}$-wave amplitude at the time of implantation, the ARVC and HCM groups did not significantly differ from the control group; (2) during the 5-year follow-up period, no significant decrease in R-wave amplitude was observed in the HCM group, but was found in the ARVC group when compared to the control group; (3) there was a significant difference in $\mathrm{R}$-wave amplitude and pacing threshold at year 5 between the ARVC and control groups; and (4) during the follow-up period, the ARVC and HCM groups showed a similar amount of change in the pacing threshold and pacing impedance compared with the control group.

A previous study analyzed cases with different lead positions such as the apex and ventricular septum. ${ }^{5}$ In contrast, in this study, all patients had the ICD lead implanted in the RV apex, and the confirmation of the lead position relied on imaging including computed tomography and fluoroscopy. This was one of the strengths of our study as there was no bias regarding the lead position.

ARVC is characterized by the progressive replacement of the right ventricle with fibrofatty tissue. This replacement is initially localized but gradually spreads throughout the entire right ventricle, and in some cases, the left ventricle is also replaced with fibrofatty tissue. However, in ARVC, the ventricular septum is rarely replaced with fibrofatty tissue. ${ }^{6-8)} \mathrm{HCM}$ is a cardiac disease characterized by abnormal hypertrophy of the left ventricular myocardium. It is classified into types such as hypertrophic obstructive cardiomyopathy, apical hypertrophic cardiomyopathy, and middle ventricular obstructive cardiomyopathy. In a few patients with HCM, it progresses to the dilated phase, in which a reduction in the left ventricular contractility and left ventricular dilatation occurs. $^{9-11)}$

Previous studies have reported that the RV apex tends to be replaced with fibrofatty tissue, which could cause worsening of the R-wave amplitude and other parameters during long-term follow-up in patients with ARVC. ${ }^{5)}$

In this study, the R-wave amplitude in the ARVC group significantly decreased compared to that in the control group, and the R-wave amplitude at the time of the last follow-up was significantly lower than that in the control group. Moreover, RV diameter was large in ARVC patients with a decline in $\mathrm{R}$ wave although there was no statistical difference, and this finding may reflect progression of the disease. The abrupt change of $\mathrm{R}$ wave amplitude between year 3 and year 5 occurred in the ARVC group, and the reason could be speculated as follows. Replacing RV with local fibrofatty tissue is known to take time. ${ }^{19)}$ The results from this study suggest that at least 3 years are needed before the RV apex was transmurally replaced with sufficient fibrofatty tissue to affect the $\mathrm{R}$ wave amplitude. However, the cause could not be identified in this study.

Excessive loop of RV lead on the tricuspid annulus could also have an influence on the later $\mathrm{R}$ wave amplitude's change, but there was no excessive loop in all
ARVC cases in this study. Some ICD models can select two modes for RV sensing; tip-ring and tip-coil. However, all ICDs in this study were models that could not switch $\mathrm{RV}$ sensing mode, so it is unlikely that the decrease in $\mathrm{R}$ wave amplitude was related to the sensing mode.

Although the pacing threshold increased and pacing impedance decreased during the 5-year period, the amount of change in them did not significantly differ. Previous studies showed that during a longer follow-up period, the pacing threshold in ARVC patients tended to have a significant increase compared with that in non-ARVC patients. ${ }^{5,20)}$ In this study, we also found no significant difference in pacing threshold at the time of implantation between the ARVC and control groups, but the threshold at the time of last follow-up in the ARVC group was significantly higher than that in the control group. Therefore, if the observation period of this study is extended further, a significant increase in pacing threshold might be obtained in the ARVC group.

Based on these findings, the intracardiac parameters of the ICD lead may deteriorate between 3 and 5 years for R-wave amplitude and 5 years or more for pacing threshold. In other words, R-wave amplitude is likely to worsen before the pacing threshold rises in the ARVC group. If the deterioration of R-wave amplitude begins clinically, it may be necessary to follow-up with a shorter interval.

In patients with $\mathrm{HCM}$, the R-wave amplitude is also reported to deteriorate during long-term follow-up. ${ }^{4)}$ However, in this study, all parameters remained unchanged in the HCM group and there were no significant differences between the HCM and control groups in terms of the transition of all parameters during the follow-up period. In this study, the observation period of 5 years may have been inadequate for a significant difference in the parameters to occur, because the incidence of development of dilated-phase HCM is reported to be about 5 per 1000 patient-years, and a much longer observation period than 5 years is needed. ${ }^{9-11)}$ In HCM patients, cardiac magnetic resonance-identified late gadolinium enhancement (CMRLGE), which represents regional fibrosis, and is usually localized at the RV septal insertion sites and in walls with the greatest hypertrophy. ${ }^{21,22)}$ Thus, the results obtained in this study may be reasonable because all ICD leads were implanted in the RV apex where a CMR-LGE is rarely found.

As noted earlier, the RV septum is not usually replaced by fibrofatty tissue and is likely to be healthy in ARVC patients. ${ }^{6-8)}$ Since the RV apex may have a risk of $\mathrm{R}$-wave deterioration, it may be advisable to make the RV septum the first choice for ICD-lead implantation in ARVC cases. However, there are limited data regarding the comparison of the RV septum and RV apex with respect to the lead location in ARVC cases, ${ }^{23)}$ therefore, further study is needed to determine whether the RV septum is optimal as an ICD-lead placement site for ARVC cases. In HCM, it seems that there is no problem with an implantation in the RV apex with regard to the lead position. However, in hypertrophic obstructive cardiomyopathy, previous studies have reported that lead implantation is desirable in an RV site where the pressure gradient is further 
decreased by pacing, ${ }^{24-26)}$ and it is necessary to judge such issues in each case.

Study Limitations: There are several limitations to this study. First, it was a retrospective observational study from a single center and the number of patients with ARVC and HCM was small. Therefore, the present findings regarding a change in the ICD-lead parameters during long-term follow-up require confirmation in larger clinical trials. Second, although this study had an observation period of 5 years, a longer follow-up period may be needed to observe a difference in pacing threshold and impedance, as well as in sensing. Finally, the diagnosis of ARVC was based on the Task Force Criteria and gene examination was rarely performed.

\section{Conclusions}

This observational study, which evaluated the ICDlead electronic parameters of different cardiomyopathies, showed that ventricular sensing is likely to deteriorate in ARVC patients but not in HCM and other non-SHD patients in the 5 years after implantation. Implantation of an ICD lead in the RV septum should be considered in ARVC patients.

\section{Acknowledgment}

We would like to thank Mr. John Martin for his linguistic assistance.

\section{Disclosure}

Conflicts of interest: The authors declare that there were no conflicts of interest.

\section{References}

1. Kuck KH, Cappato R, Siebels J, Ruppel R. Randomized comparison of antiarrhythmic drug therapy with implantable defibrillators in patients resuscitated from cardiac arrest: the Cardiac Arrest Study Hamburg (CASH). Circulation 2000; 102: 748-54.

2. Bokhari F, Newman D, Greene M, Korley V, Mangat I, Dorian P. Long-term comparison of the implantable cardioverter defibrillator versus amiodarone: eleven-year follow-up of a subset of patients in the Canadian Implantable Defibrillator Study (CIDS). Circulation 2004; 110: 112-6.

3. Hsu SS, Mohib S, Schroeder A, Deger FT. T wave oversensing in implantable cardioverter defibrillators. J Interv Card Electrophysiol 2004; 11: 67-72.

4. Watanabe H, Chinushi M, Izumi D, et al. Decrease in amplitude of intracardiac ventricular electrogram and inappropriate therapy in patients with an implantable cardioverter defibrillator. Int Heart J 2006; 47: 363-70.

5. Mugnai G, Tomei R, Dugo C, Tomasi L, Morani G, Vassanelli C. Implantable cardioverter-defibrillators in patients with arrhythmogenic right ventricular cardiomyopathy: the course of electronic parameters, clinical features, and complications during long-term follow-up. J Interv Card Electrophysiol 2014; 41: 23-9

6. Marcus FI, Fontaine GH, Guiraudon G, et al. Right ventricular dysplasia: a report of 24 adult cases. Circulation 1982; 65: 384 98.
7. Thiene G, Nava A, Corrado D, Rossi L, Pennelli N. Right ventricular cardiomyopathy and sudden death in young people. $\mathrm{N}$ Engl J Med 1988; 318: 129-33.

8. Fontaine G, Fontaliran F, Frank R. Arrhythmogenic right ventricular cardiomyopathies: clinical forms and main differential diagnoses. Circulation 1998; 97: 1532-5.

9. Thaman R, Gimeno JR, Murphy RT, et al. Prevalence and clinical significance of systolic impairment in hypertrophic cardiomyopathy. Heart 2005; 91: 920-5.

10. Biagini E, Coccolo F, Ferlito M, et al. Dilated-hypokinetic evolution of hypertrophic cardiomyopathy: prevalence, incidence, risk factors, and prognostic implications in pediatric and adult patients. J Am Coll Cardiol 2005; 46: 1543-50.

11. Harris KM, Spirito P, Maron MS, et al. Prevalence, clinical profile, and significance of left ventricular remodeling in the endstage phase of hypertrophic cardiomyopathy. Circulation 2006; 114: $216-25$

12. McKenna WJ, Thiene G, Nava A, et al. Diagnosis of arrhythmogenic right ventricular dysplasia/cardiomyopathy. Task Force of the Working Group Myocardial and Pericardial Disease of the European Society of Cardiology and of the Scientific Council on Cardiomyopathies of the International Society and Federation of Cardiology. Br Heart J 1994; 71: 215-8.

13. Marcus FI, McKenna WJ, Sherrill D, et al. Diagnosis of arrhythmogenic right ventricular cardiomyopathy/dysplasia: proposed modification of the task force criteria. Circulation 2010; 121: 1533-41.

14. Nagueh SF, Bierig SM, Budoff MJ, et al. American Society of Echocardiography clinical recommendations for multimodality cardiovascular imaging of patients with hypertrophic cardiomyopathy: Endorsed by the American Society of Nuclear Cardiology, Society for Cardiovascular Magnetic Resonance, and Society of Cardiovascular Computed Tomography. J Am Soc Echocardiogr 2011; 24: 473-98.

15. Elliott PM, Anastasakis A, Borger MA, et al. 2014 ESC Guidelines on diagnosis and management of hypertrophic cardiomyopathy: the Task Force for the Diagnosis and Management of Hypertrophic Cardiomyopathy of the European Society of Cardiology (ESC). Eur Heart J 2014; 35: 2733-79.

16. Maron BJ, Ommen SR, Semsarian C, Spirito P, Olivotto I, Maron MS. Hypertrophic cardiomyopathy: present and future, with translation into contemporary cardiovascular medicine. J Am Coll Cardiol 2014; 64: 83-99.

17. McGavigan AD, Roberts-Thomson KC, Hillock RJ, Stevenson IH, Mond HG. Right ventricular outflow tract pacing: radiographic and electrocardiographic correlates of lead position. Pacing Clin Electrophysiol 2006; 29: 1063-8.

18. Suzuki T, Fujino T, Shinohara M, et al. Right ventricular septal pacing using a thin lumenless pacing lead and delivery system with a deflectable catheter. Int Heart J 2018; 59: 1253-60.

19. Basso C, Corrado D, Marcus FI, Nava A, Thiene G. Arrhythmogenic right ventricular cardiomyopathy. Lancet 2009; 373: 1289-300.

20. Link MS, Wang PJ, Haugh CJ, et al. Arrhythmogenic right ventricular dysplasia: clinical results with implantable cardioverter defibrillators. J Interv Card Electrophysiol 1997; 1: 41-8.

21. Moon JC, McKenna WJ, McCrohon JA, Elliott PM, Smith GC, Pennell DJ. Toward clinical risk assessment in hypertrophic cardiomyopathy with gadolinium cardiovascular magnetic resonance. J Am Coll Cardiol 2003; 41: 1561-7.

22. Chen X, Zhao T, Lu M, et al. The relationship between electrocardiographic changes and CMR features in asymptomatic or mildly symptomatic patients with hypertrophic cardiomyopathy. Int J Cardiovasc Imaging 2014; 30(Suppl 1): 55-63.

23. Schinkel AF. Implantable cardioverter defibrillators in arrhythmogenic right ventricular dysplasia/cardiomyopathy: patient outcomes, incidence of appropriate and inappropriate interventions, and complications. Circ Arrhythm Electrophysiol 2013; 6: 5628.

24. Minami Y, Kajimoto K, Kawana M, Hagiwara N, Sherrid MV. 
Synergistic effect of dual chamber pacing and disopyramide in obstructive hypertrophic cardiomyopathy. Int J Cardiol 2010; 141: 195-7.

25. Lucon A, Palud L, Pavin D, et al. Very late effects of dual chamber pacing therapy for obstructive hypertrophic cardio- myopathy. Arch Cardiovasc Dis 2013; 106: 373-81.

26. Yue-Cheng H, Zuo-Cheng L, Xi-Ming L, et al. Long-term follow-up impact of dual chamber pacing on patients with hypertrophic obstructive cardiomyopathy. Pacing Clin Electrophysiol 2013; 36: 86-93. 